\title{
Dynamic Analysis of ITER Tokamak Using Simplified Model for Support Structure
}

\author{
TAKEDA Nobukazu and SHIBANUMA Kiyoshi \\ Japan Atomic Energy Research Institute, Naka-machi, Naka-gun, Ibaraki 311-019, Japan \\ (Received 6 September 2004 / Accepted 12 October 2004)
}

\begin{abstract}
The simplified analytical model of a support structure composed of complicated structures such as multiple flexible plates was proposed for dynamic analysis of the ITER major components of the VV and TF coil. The support structure composed of flexible plates and connection bolts was modeled as a spring model composed of only two spring elements simulating the in-plane and out-of-plane stiffness of the support structure with flexible plates including the effect of connection bolts. The stiffness of both these spring models for the VV and TF coil agree well with that of shell models simulating actual structures such as flexible plates and connection bolts. It is therefore found that the spring model with only these two parameters, in-plane and out-of-plane stiffness, simplifies the complicated support structure with flexible plates for the dynamic analysis of the VV and TF coil. Using the proposed spring model, the dynamic analysis of the VV and TF coil for the ITER were performed to estimate the integrity under the design earthquake at Rokkasho, a candidate for an ITER site. As a result, it is found that the maximum relative displacement of $8.6 \mathrm{~mm}$ between the $\mathrm{VV}$ and TF coil is much less than the current design gap of $100 \mathrm{~mm}$, so that the integrity of the major components (VV and TF coil) of the ITER tokamak device is ensured for the expected earthquake event.
\end{abstract}

\section{Keywords:}

ITER, support structure, flexible plate, dynamic analysis, spring model, simplified model, earthquake, seismic response

\section{Introduction}

In the design of integrated plants such as the ITER, one of the issues is displacement of major components caused by an earthquake. If the relative displacement of the components becomes large and exceeds the initial gap between them, they might be damaged by collision. In general, the dynamic behavior of the component is strongly affected by the mechanical features of the support structure. Typically, the ITER adopts an assembly of flexible plates as the support structure of major components such as vacuum vessel (VV) and the toroidal field coil (TF coil) in order to permit thermal deformation in the radial direction that results from the change in operation temperatures from room temperature to $200^{\circ} \mathrm{C}$ for $\mathrm{VV}$ and from room temperature to $4 \mathrm{~K}$ for the $\mathrm{TF}$ coil [1] (see Fig. 1). Thus, the stiffness of the support structure with flexible plates is relatively low in the out-of plane direction and high in the in-plane direction, so that the support structures of the VV and TF coil strongly affect the dynamic behaviors. Dynamic analysis is therefore required to estimate the precise displacement of the VV and TF coil during earthquake conditions in order to ensure the integrity of the ITER. The ITER is however composed of 18 support structures for the TF coil as well as 18 support structures for $\mathrm{VV}$, arranged in the toroidal direction as shown in Fig. 1. In addition, the support structure is composed of multi-plates, flanges, and connection bolts. For example, the TF coil support structure is composed of 21 trapezoidal flexible

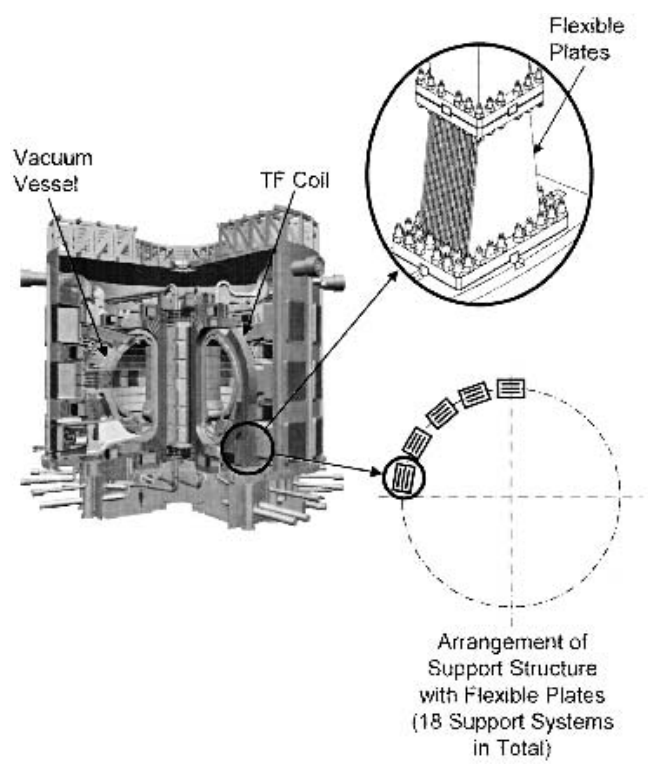

Fig. 1 An example of support structure using flexible plate for ITER. 
plates, whose dimensions are $1.035 \mathrm{~m}$ in upper width, $1.5 \mathrm{~m}$ in lower width, $2.25 \mathrm{~m}$ in height, and $30 \mathrm{~mm}$ in thickness, respectively. The support structure is connected by 36 bolts with a nominal diameter of $100 \mathrm{~mm}$ (M100) on the tokamak base structure and 20 bolts with a nominal diameter of 80 $\mathrm{mm}(\mathrm{M} 80)$ at the bottom of the TF coil through the flanges, respectively. Modeling the support structure is therefore the most critical issue for dynamic analysis of the ITER due to complication of the precise modes of the support structures of the VV and TF coil.

Based on the above reasons, the present paper proposes a simplified model of the support structures with flexible plates for the VV and TF coil. Using the proposed model, dynamic analysis is performed under the design earthquake assumed at Rokkasho, a candidate site for the ITER.

\section{Modal Analysis using Shell Model}

Modal analyses were performed using a model of a vibration system composed of a dummy weight and four support structures with flexible plates. The four support structures were placed on a circle with the same radius as the real machine, at angular intervals of 90 degrees, as shown in Fig. 2. Using this model, a parameter survey was performed by changing the values of the angle between out-of-plane (low stiffness) and vibration directions of the support structures in order to estimate the dependence of horizontal stiffness of the support structure on the vibration direction.

The support structure was modeled with shell elements for flexible plates and beam elements for bolts fixing the support structure on the base and the component. In the analysis model, the stiffness of the bolts was assumed as finite in the axial direction only; it was infinite in the other directions. This assumption can be realized and ensured by adopting a mechanism such as a shear key to prohibit horizontal slippage between connection interfaces of the support structure.

The horizontal stiffness of the support structure was calculated from the eigen frequency of the swaying vibration mode. The calculated stiffness of the VV and TF coil support structures at the angles $\theta(0,25,45,65$, and 90 degrees $)$ of the flexible plates to the vibration direction are indicated by the symbols of circles and squares in Fig. 3, respectively. From these results, a spring model of the support structure was proposed to simplify the dynamic analysis model. This spring model is composed of only two spring elements simulating the in-plane and out-of-plane stiffness of the support structure with flexible plates including the effect of connection bolts. The vertical stiffness of the spring model can be modeled as infinite. This is because (1) the primary purpose of the dynamic analysis is to estimate the horizontal deformation of the VV and TF coil caused by the horizontal vibration, and (2) the effect of the vertical stiffness by bolt connection on the horizontal vibration is already included in the calculated horizontal stiffness. The values of in-plane and out-of-plane stiffness of the spring element were directly obtained from the results at 90 and 0 degrees as shown in
Fig. 3, respectively. Table 1 shows the in-plane (90 degrees) and out-of-plane ( 0 degrees) eigen frequencies $f_{\text {in-plane }}$ and $f_{\text {out-of-plane }}$ for the VV and TF coil obtained by the shell model, respectively. The values $K_{\text {in-plane }}$ and $K_{\text {out-of-plane }}$ of stiffness used for the spring models for the VV and TF coil are also summarized in Table 1, respectively. The angular dependency of the support structure stiffness $K_{\text {spring }}$ of the spring model can be expressed as follows with $K_{\text {in-plane }}, K_{\text {out-of-plane }}$ :

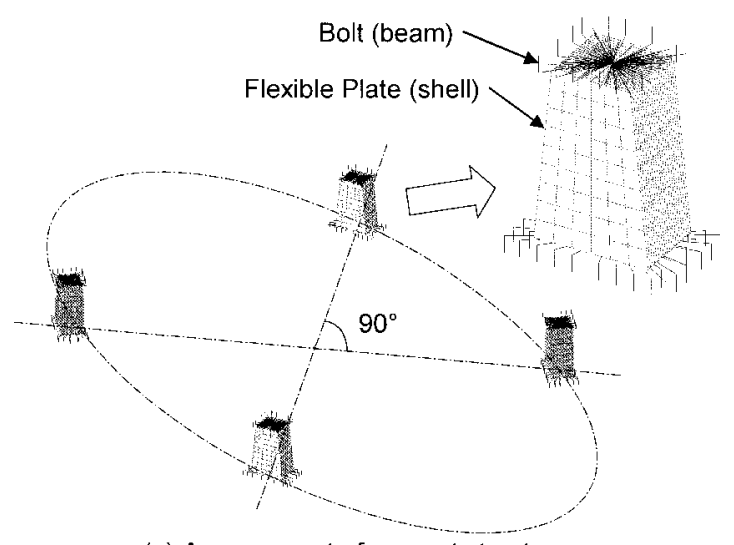

(a) Arrangement of support structures

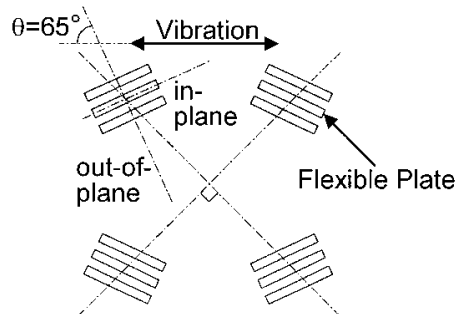

(b) Schematic plan view

Fig. 2 Example of Vibration System for TF Coil (65 degree).

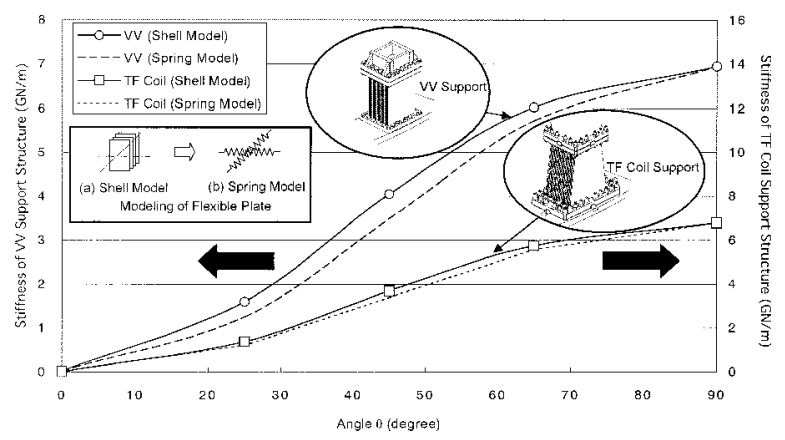

Fig. 3 Comparison of Support Stiffness between Analysis Models.

Table 1 Eigen Frequency Obtained by Shell Model and Stiffness of Support Structure Used for Spring Model.

\begin{tabular}{|l|c|c|c|c|c|}
\hline & Weight & $\begin{array}{c}f_{\text {in-plane }} \\
(90 \text { degrees })\end{array}$ & $\begin{array}{c}f_{\text {out-of-plane }} \\
(0 \text { degree })\end{array}$ & $\begin{array}{c}K_{\text {in-plane }} \\
(90 \text { degrees })\end{array}$ & $\begin{array}{c}K_{\text {out-of-plane }} \\
(0 \text { degree })\end{array}$ \\
\hline $\begin{array}{l}\text { Vacuum } \\
\text { Vessel }\end{array}$ & 9,300 ton & $8.7 \mathrm{~Hz}$ & $0.48 \mathrm{~Hz}$ & $7.0 \mathrm{GN} / \mathrm{m}$ & $21 \mathrm{MN} / \mathrm{m}$ \\
\hline $\begin{array}{l}\text { Toroidal } \\
\text { Field } \\
\text { Coii }\end{array}$ & 9,370 ton & $8.6 \mathrm{~Hz}$ & $0.37 \mathrm{~Hz}$ & $6.8 \mathrm{GN} / \mathrm{m}$ & $13 \mathrm{MN} / \mathrm{m}$ \\
\hline
\end{tabular}




$$
K_{\text {spring }}=K_{\text {in-plane }} \sin ^{2} \theta+K_{\text {out-of-plane }} \cos ^{2} \theta
$$

The results are shown as a broken line for the VV and a dotted line for TF coil in Fig. 3. The stiffness of both spring models for the VV and TF coil agree well with that of shell models although the stiffness of both spring models is slightly lower than that of the shell models. It is therefore found that the spring model with only two parameters, in-plane and out-ofplane stiffness, enables us to simplify the complicated support structure with flexible plates for the dynamic analysis of the VV and TF coil with slightly safer side estimation.

\section{Dynamic Analysis using a Spring Model}

As mentioned above, the horizontal displacement of the $\mathrm{VV}$ and TF coil of the ITER is the primary concern in an earthquake. Therefore, dynamic analyses of the VV and TF coil were performed in order to estimate the relative displacement in the horizontal direction during such an event. Analytical models for the VV and TF coil are shown in Fig. 4. Based on the results in the previous section, the spring models were adopted for the VV and the TF coil support structures.

The input acceleration wave for the dynamic analysis of the VV and TF coil was calculated based on the response analysis of the whole tokamak building [2]. The input earthquake motion for the analysis of the building was 0.45 $\mathrm{m} / \mathrm{s}$ in velocity at the base stratum [2]. This is almost the same as the maximum design seismic wave used for the nuclear facility in Rokkasho area, a candidate site for the ITER.

Figure 5 shows typical deformation results of the VV and TF coil under the dynamic analysis, respectively. The maximum horizontal displacements of the VV and TF coil were $4.5 \mathrm{~mm}$ at the point $\mathrm{A}$ and $4.1 \mathrm{~mm}$ at the point $\mathrm{B}$ as shown in Fig. 5, respectively. The VV and the TF coil faced each other across a gap of $100 \mathrm{~mm}$. Point A of VV and point B of TF coil sat directly opposite each other on either side of this gap. The maximum relative displacement between the VV and TF coil can therefore be estimated as a sum of their maximum displacements. It is therefore found that the maximum relative displacement of $8.6 \mathrm{~mm}$ between $\mathrm{VV}$ and TF coil is much less than $100 \mathrm{~mm}$, so that the integrity of the major components (VV and TF coil) of the ITER tokamak device are ensured for the earthquake event.

\section{Conclusion}

The simplified analytical model of the support structure with multiple flexible plates was proposed for the dynamic analysis of VV and TF coil as the ITER major components. The support structure with flexible plates and bolt connection was modeled as a spring model composed of only two spring elements simulating the in-plane and out-of-plane stiffness of the support structure with flexible plates including the effect of connection bolts. The stiffness of both spring models for the VV and TF coil agrees well with that of shell models although the stiffness of both spring models is slightly lower

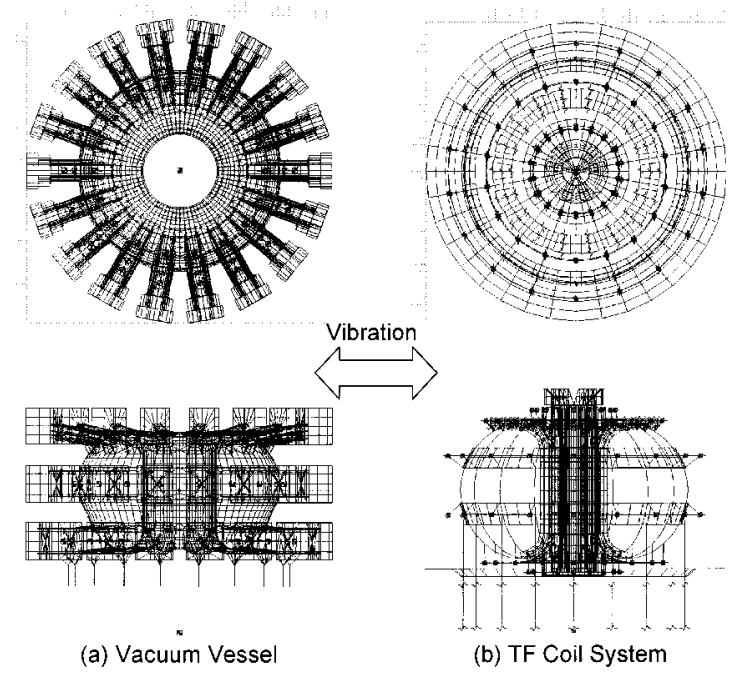

Fig. 4 Analytical Model.

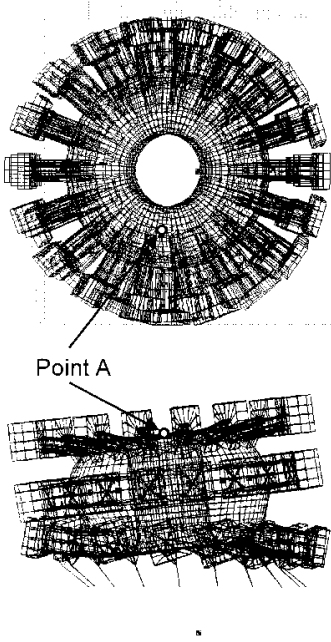

(a) Vacuum Vessel

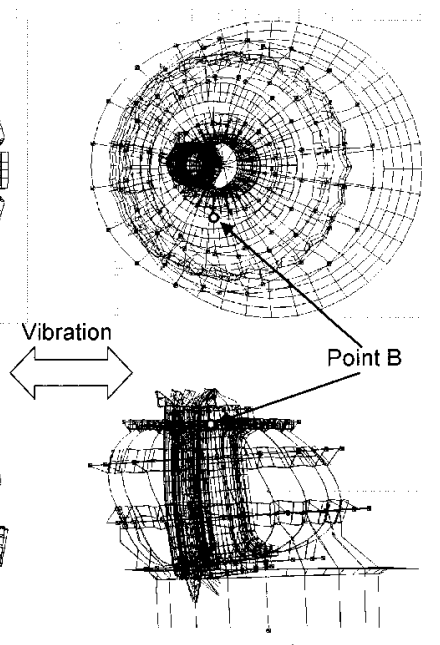

(b) TF Coil System
Fig. 5 Deformation and Estimation Points.

than that of the shell models. It is therefore found that the spring model with only two parameters, in-plane and out-ofplane stiffness, enables us to simplify the complicated support structure with flexible plates for the dynamic analysis of the VV and TF coil with slightly safer side estimation. Using the simplified spring model, the dynamic analysis of the VV and TF coil for the ITER were performed for estimation under the design earthquake at Rokkasho area. As a result, it is found that the maximum relative displacement of $8.6 \mathrm{~mm}$ between VV and TF coil is much less than the current design gap of $100 \mathrm{~mm}$, so that the integrity of the major components (VV and TF coil) of the ITER tokamak device are ensured for the earthquake event.

\section{References}

[1] ITER Technical Basis, ITER EDA Document Series No. 24, IAEA, Vienna, 2002.

[2] N. Takeda et al., to be published in J. Jpn. Association for Earthquake Engineering, Vol. 4, No. 3 (2004). 